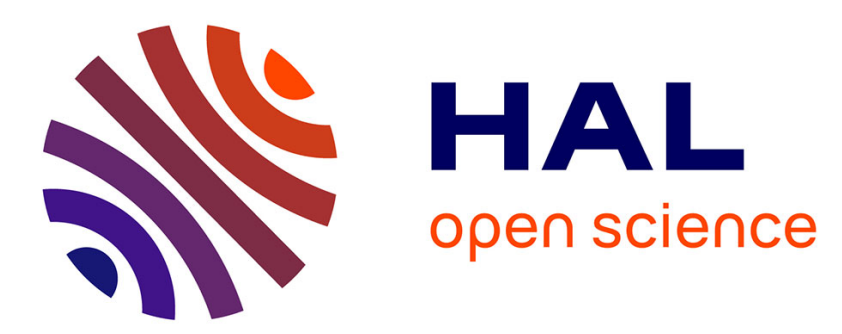

\title{
Direct Transition from Bose Glass to Normal State in the $(\mathrm{K}, \mathrm{Ba}) \mathrm{BiO} 3$ Superconductor
}

Thierry Klein, C. Marcenat, S. Blanchard, J. Marcus, C. Bourbonnais, R. Brusetti, C. J. van Der Beek, M. Konczykowski

\section{- To cite this version:}

Thierry Klein, C. Marcenat, S. Blanchard, J. Marcus, C. Bourbonnais, et al.. Direct Transition from Bose Glass to Normal State in the (K,Ba)BiO3 Superconductor. Physical Review Letters, 2004, 92, pp.037005. 10.1103/PhysRevLett.92.037005 . hal-00959094

\section{HAL Id: hal-00959094 https://hal.science/hal-00959094}

Submitted on 13 Mar 2014

HAL is a multi-disciplinary open access archive for the deposit and dissemination of scientific research documents, whether they are published or not. The documents may come from teaching and research institutions in France or abroad, or from public or private research centers.
L'archive ouverte pluridisciplinaire HAL, est destinée au dépôt et à la diffusion de documents scientifiques de niveau recherche, publiés ou non, émanant des établissements d'enseignement et de recherche français ou étrangers, des laboratoires publics ou privés. 


\title{
Direct Transition from Bose Glass to Normal State in the $(\mathrm{K}, \mathrm{Ba}) \mathrm{BiO}_{3}$ Superconductor
}

\author{
T. Klein,,${ }^{1,2}$ C. Marcenat, ${ }^{3,4}$ S. Blanchard, ${ }^{1}$ J. Marcus,${ }^{1}$ C. Bourbonnais, ${ }^{4}$ R. Brusetti, ${ }^{5}$ \\ C. J. van der Beek, ${ }^{6}$ and M. Konczykowski ${ }^{6}$ \\ ${ }^{1}$ Laboratoire d'Etudes des Propriétés Electroniques des Solides, CNRS, BP 166, 38042 Grenoble CEDEX 9, France \\ ${ }^{2}$ Institut Universitaire de France and Université Joseph Fourier, BP 53, 38041 Grenoble CEDEX 9, France \\ ${ }^{3}$ Commissariat à l'Energie Atomique-Grenoble, Département de Recherche Fondamentale sur la Matière Condensée, SPSMS, \\ 17 rue des Martyrs, 38054 Grenoble CEDEX 9, France \\ ${ }^{4}$ Département de physique, Université de Sherbrooke, 2500 Boulevard Université, Sherbrooke, Quebec, Canada JIK $2 R 1$ \\ ${ }^{5}$ Centre de Recherche sur les Très Basses Températures, Centre National de la Recherche Scientifique, \\ BP 166, 38042 Grenoble CEDEX 9, France \\ ${ }^{6}$ Laboratoire des Solides Irradiés, CNRS-UMR 7642 and CEA/DSM/DRECAM, Ecole Polytechnique, \\ 91128 Palaiseau CEDEX, France \\ (Received 15 May 2003; published 23 January 2004)
}

\begin{abstract}
The introduction of columnar defects in $(\mathrm{K}, \mathrm{Ba}) \mathrm{BiO}_{3}$ single crystals shifts both the irreversibility and thermodynamic transition lines, respectively, deduced from ac susceptibility (and/or transport) and specific heat measurements, upwards. This shift can be attributed to the defect-induced decrease of the difference $(\Delta F)$ between the free energies in the superconducting and the normal states, assuming that the position of the superconducting transition is given by the condition $|\Delta F| \approx k_{B} T / \xi^{3}$. This criterion also perfectly reproduces the influence of the angle between the tracks and the external field. This result suggests that no vortex liquid phase exists in this system.
\end{abstract}

DOI: 10.1103/PhysRevLett.92.037005

PACS numbers: 74.25.Bt, 74.25.Dw, 74.25.Op

The $(B-T)$ phase diagram of high $T_{c}$ cuprates has been the topic of a large amount of theoretical and experimental work. One of the most important discoveries has been the first order melting of the flux line lattice to a vortex liquid phase [1] due to strong thermal fluctuations, enhanced by the combination of high critical temperatures and large crystalline anisotropies.

The strong upward curvature of the irreversibility line $B_{\text {irr }}(T)$ (IRL), determined from the (critical) scaling properties of the current-voltage $(I-V)$ characteristics (and/or from the onset of harmonics in ac-susceptibility measurements), suggests that a vortex liquid phase also exists in the $(\mathrm{K}, \mathrm{Ba}) \mathrm{BiO}_{3}(\mathrm{KBBO})$ compound, in spite of its cubic structure and its moderate $T_{c} \sim 30 \mathrm{~K}$. Both in cuprates [2,3] and KBBO [4], $B_{\text {irr }}(T)$ progressively shifts towards higher values when increasing the density of amorphous columnar defects by successive irradiation with swift heavy ions. Together with its dependence on the orientation of the magnetic field relative to the ion tracks and the scaling of the $I-V$ characteristics, this seemed to justify the identification of $B_{\text {irr }}(T)$ with the transition from the so-called Bose glass [5] to the vortex liquid. However, we have recently shown that the main thermodynamic transition line $B_{C_{p}}(T)$ deduced from specific heat measurements also presents a concave curvature [6] and is shifted together with $B_{\text {irr }}(T)$ following heavyion irradiation (HII) [7]. The possible influence of local superconductivity along the tracks or mean-free path effects on the transition has been ruled out in Ref. [7] so that the origin of this behavior remained an open question.
In this Letter, we present a complete investigation of the influence of the irradiation dose and the relative orientation $\theta$ between the tracks and the external field on the superconducting transition. The location of the $B_{C_{p}}(T, \theta)$ and $B_{\text {irr }}(T, \theta)$ lines can be very well reproduced by a field-dependent criterion [8], $|\Delta F(H)|=\left|F_{n}-F_{s}(H)\right|=$ $\alpha k_{B} T / \xi^{3}$. Here $F_{s}(H)$ and $F_{n}$ are the free energy densities in the superconducting state with quenched disorder (i.e., the pinned vortex solid) and in the normal state, $\xi(T)$ is the Ginzburg-Landau (GL) coherence length, and $\alpha$ is a constant. Such a criterion is often used to estimate the width of the critical region, but we show below that the temperature calculated from this criterion actually coincides with the location of the jump in $C_{p}$ at the onset of superconductivity. In other words, due to thermal fluctuations, the field $B^{*}(T)$ at which the superconducting transition occurs is significantly smaller than the mean-field upper critical field $B_{c 2}^{\mathrm{MF}}=\Phi_{0} / 2 \pi \xi^{2}$. The effect of vortex localization on the ion tracks is to lower $F_{s}(H)$, thereby shifting the superconducting transition to higher temperature. These results impose a new interpretation of the $(B-T)$ phase diagram of $\mathrm{KBBO}$, in which no vortex liquid phase exists but in which the vortex solid directly "sublimates" into the normal state.

The ac susceptibility and specific heat measurements were performed on $\mathrm{HII}(\mathrm{K}, \mathrm{Ba}) \mathrm{BiO}_{3}$ single crystals with dose-equivalent matching fields (at which the track density is equal to the vortex density $\Phi_{0} / B$ ) of $B_{\Phi}=1,2$, and $6 \mathrm{~T}$. The irradiation, with $7.2 \mathrm{GeV} \mathrm{Ta}^{57+}$ ions, was performed at the Grand Accélérateur National d'Ions Lourds in Caen, France. Further details on the experi- 
mental procedure can be found in Refs. [4,6,7]. The critical temperatures $T_{c}$ of the different crystals ranged from $31.5 \mathrm{~K}$ to about $31.9 \mathrm{~K}$. In what follows, all $T_{c}$ values have been scaled to $31.5 \mathrm{~K}$ for a better comparison between crystals. At $T_{c}, C_{p}$ is dominated by the phonon contribution and the superconducting jump is only on the order of $5 \times 10^{-3}$ of the total specific heat. Therefore, Fig. 1(a) displays only the temperature dependence of the difference between the specific heat in the superconducting and normal states $\left[\Delta C_{p}=C_{p}(H, T)-C_{p}(7 \mathrm{~T}, T)\right]$ for various irradiation doses. Indeed, the baseline $C_{p}(7 \mathrm{~T}, T)$ represents the normal state specific heat in the temperature range of the figure. The specific heat jump is progressively shifted towards higher temperatures with increasing irradiation dose, in the same way as the IRL [4]. Moreover, the specific heat jump moves back to lower temperatures when the magnetic field is tilted away from the track direction [see Fig. 1(b)], finally coinciding with the transition measured in the pristine sample when the field is applied perpendicularly to the tracks (see also [7]).

Generalizing the approach proposed by Cooper $e t$ al. [8] to the case of irradiated samples, we obtain a characteristic field $B^{*}$ by solving

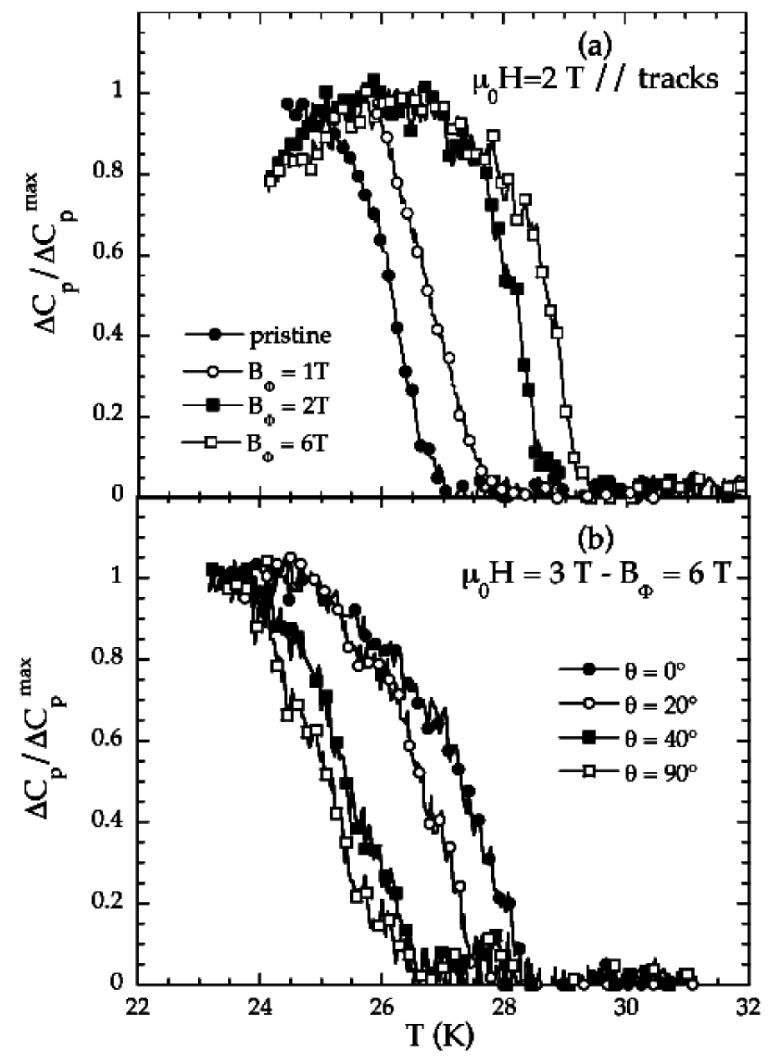

FIG. 1. Temperature dependence of the magnetic field dependent contribution to the specific heat at (a) $B=2 \mathrm{~T}$ for various irradiation doses ( $H$ being parallel to the tracks) and (b) $B=$ $3 \mathrm{~T}$ and $B_{\Phi}=6 \mathrm{~T}$ for various angles between the tracks and the external field.

$$
\Delta F=\Delta F_{0}-n_{t}\left(B^{*}\right) U_{p}\left(1-B^{*} / B_{c 2}\right)=\alpha k_{B} T / \xi^{3},
$$

where $\Delta F_{0}=-B_{c}^{2} / \mu_{0}\left(1-B^{*} / B_{c 2}\right)^{2}, \quad B_{c}^{2} / 2 \mu_{0}=F_{n}-$ $F_{s}(0)$ is the condensation energy, $n_{t}(B)=\left(B_{\Phi} / \Phi_{0}\right) \times$ $\left[1-\exp \left(-\beta B_{\Phi} / B\right)\right] /\left(\beta B_{\Phi} / B\right) f\left(T / T_{d p}\right)$ is the number of trapped vortices [9], $U_{p}$ is the pinning energy per unit length, $\beta=2 \xi /\left(c_{0}+\xi\right)$ (with $c_{0}$ the track radius), $f(x)$ is a thermal smearing function [1], and $T_{d p}(T)$ is the depinning energy. The depression of the order parameter due to the proximity of the mean-field $B_{c 2}$ line is taken into account by the factor $\left(1-B^{*} / B_{c 2}\right)$. From Eq. (1), one directly sees that the decrease of the free energy by irradiation implies an increase of $B^{*}$. However, we show below that, more than being just qualitative, this simple model provides a very good quantitative description of the location of the specific heat jump after irradiation, justifying the identification of $B^{*}$ with the superconducting transition field. This a posteriori confirms the assumption previously made by the authors of Ref. [8] (in crystals without columnar defects), who only compared $B^{*}$ to nonthermodynamic resistive transition fields.

The transition temperatures deduced from specific heat measurements (open symbols) in a pristine and irradiated samples are displayed in Fig. 2 together with $B^{*}(T)$ deduced from Eq. (1) (closed symbols). As shown, the model very well reproduces the progressive shift of the superconducting transition line with increasing irradiation dose. Here, we have determined the coherence

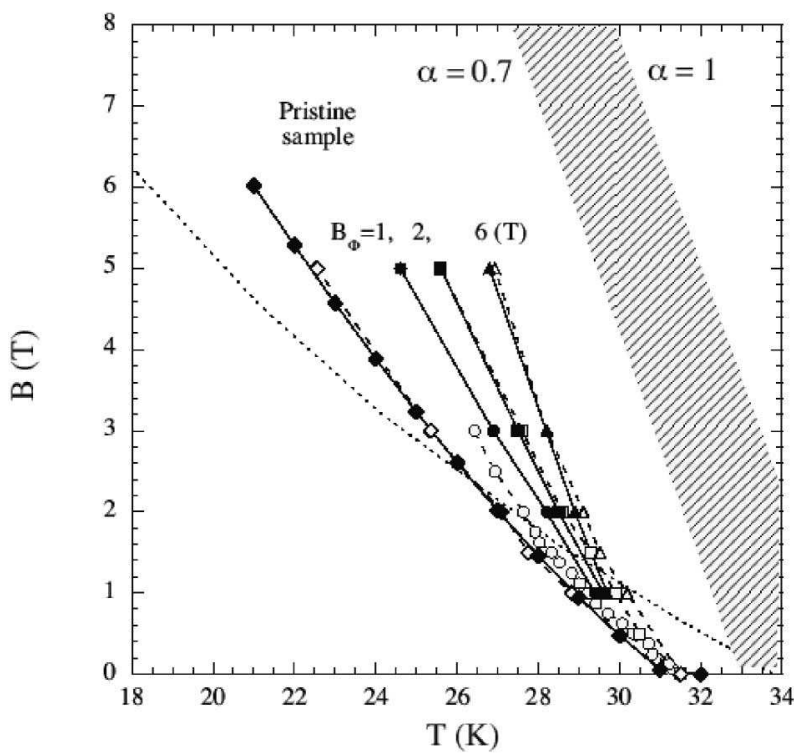

FIG. 2. $B-T$ phase diagram of a pristine (diamonds) and irradiated $\mathrm{KBBO}$ samples with $B_{\Phi}=1 \mathrm{~T}$ (circles), $B_{\Phi}=2 \mathrm{~T}$ (squares), and $B_{\Phi}=6 \mathrm{~T}$ (triangles) showing good agreement between the experimental data (open symbols) deduced from specific heat measurements and the values predicted from Eq. (1) (closed symbols). The dotted line is the prediction derived from Eq. (2) and the hatched area indicates the location of the mean field transition for $0.7 \leq \alpha \leq 1$ (see text for details). 
length, $\xi \approx 32 \pm 2 \AA$, from the low temperature upper critical field $\left(B_{c 2}(0) \sim 30 \mathrm{~T}\right)$. The penetration depth $\lambda(0) \approx 3000-3500 \AA$ can be estimated from neutron scattering data [10], while $U_{p}=c \varepsilon_{1}$, with $\varepsilon_{1}$ the vortex line tension and $c \approx 0.7-0.8$ (see below). The only free parameters are the mean field critical temperature $\left(T_{c}^{\mathrm{MF}}\right)$ and $\alpha$. The determination of $\alpha$ would require some microscopic model but, as previously obtained by [8], we assume that $\alpha$ is of order unity. As an example, the values reported on Fig. 2 have been calculated taking $\alpha=1$ which yields $T_{c}^{\mathrm{MF}} \approx 35-36 \mathrm{~K}$ but very similar values can be obtained by slightly reducing $\alpha$ to $\approx 0.7$ which brings $T_{c}^{\mathrm{MF}}$ down to $\approx 33 \mathrm{~K}$.

In the Bose glass melting scenario, the shift of the melting temperature $\left[B_{\mathrm{BG}}(T)\right]$ with irradiation dose can be estimated in the so-called single-line-many-defects regime (in which individual flux lines are weakly pinned by many columns) to $d B_{\mathrm{BG}} / d T \propto 1+A B_{\Phi}^{1 / 2}$. This dependence is well verified in both cuprates [2] and $(\mathrm{K}, \mathrm{Ba}) \mathrm{BiO}_{3}$ [4]. However, the coefficient $A$ is about twice smaller in $(\mathrm{K}, \mathrm{Ba}) \mathrm{BiO}_{3}$ than in $\mathrm{YBa}_{2} \mathrm{Cu}_{3} \mathrm{O}_{7-\delta}$, whereas one would expect it to be about 20 times larger [4]. On the other hand, our model, with the same parameters as above, provides very good quantitative agreement with the experimental data and, as shown in the inset of Fig. 3, predicts an evolution of $d B^{*} / d T$ with irradiation dose that closely matches the empirical $B_{\Phi}^{1 / 2}$ law.

Another striking feature is the "matching field effect" which shows up in the irreversibility line as a kink for

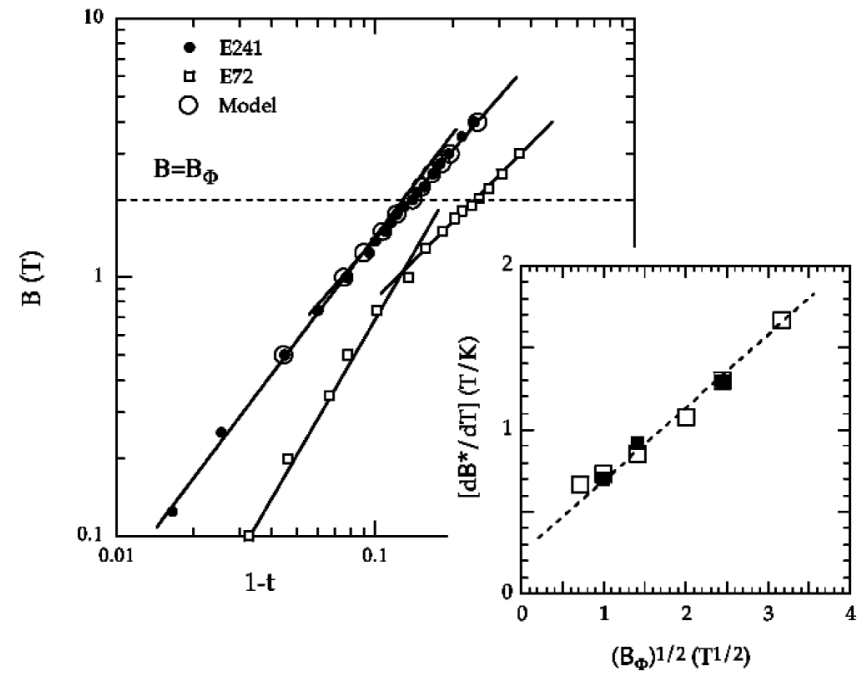

FIG. 3. Irreversibility field as a function of $1-t$ (where $t=T / T_{c}$ ) in the $B_{\Phi}=2 \mathrm{~T}$ irradiated sample (solid circle) compared with the calculated values (open circles). The "matching field effect" is better visible in the overdoped sample which contains more point defects (open squares, from [4]). Inset: Dependence of the linear slope of the transition line deduced from specific heat measurements on $B_{\Phi}$ for $(\mathrm{K}, \mathrm{Ba}) \mathrm{BiO}_{3}$ (solid squares) as compared to the values deduced from the model (open squares).
$B \approx B_{\Phi}$ [4]. In the Bose glass melting scenario, the presence of ever more unpinned interstitial vortices for $B>B_{\Phi}$ is expected to drive progressively the IRL of the irradiated sample towards that of the pristine sample. This effect also occurs in our model as $n_{t}$ saturates at high fields. In the present sample, this effect is rather small but in perfect agreement with what the model predicts [11] (open circles in Fig. 3, this effect is best visible when $B_{\text {irr }}$ is plotted as a function of $1-T / T_{c}$ ). A much larger effect was observed in an overdoped sample $\left(T_{c} \sim 21 \mathrm{~K}\right)$ for which the IRL already starts to bend over for $B>B_{\Phi} / 2$. This latter sample, however, is much more inhomogeneous and the presence of a larger matching field effect is presumably due to a large amount of point defects, which are not taken into account here.

We finally discuss the influence of the angle $\theta$ between the tracks and the direction of the external field on the position of the transition line. Let $r, s$, and $t$ be the average length of the vortex segments trapped on the tracks, of the vortex sections joining tracks, and of a hypothetical vortex section following the average field direction, respectively (see inset of Fig. 4). The pinning energy per unit length $U_{p}$ in Eq. (1) can then be recalculated as $U_{p}(\theta)=\left[r U_{p}-(r+s-t) \varepsilon_{1}\right] /(r+s)$ [1]. Minimizing this expression with respect to $r$ at a fixed angle leads to $s / d=\left[1-\left(1-U_{P} / \varepsilon_{1}\right)^{2}\right]^{-1 / 2}(d$ is the average distance between tracks) and

$$
U_{p}(\theta)=U_{p}\left[1-\frac{\frac{2 s}{d} \sin \theta+\frac{\varepsilon_{1}}{U_{p}}(\cos \theta-1)}{\cos \theta+\frac{U_{P}}{\varepsilon_{1}} \frac{s}{d} \sin \theta}\right] .
$$

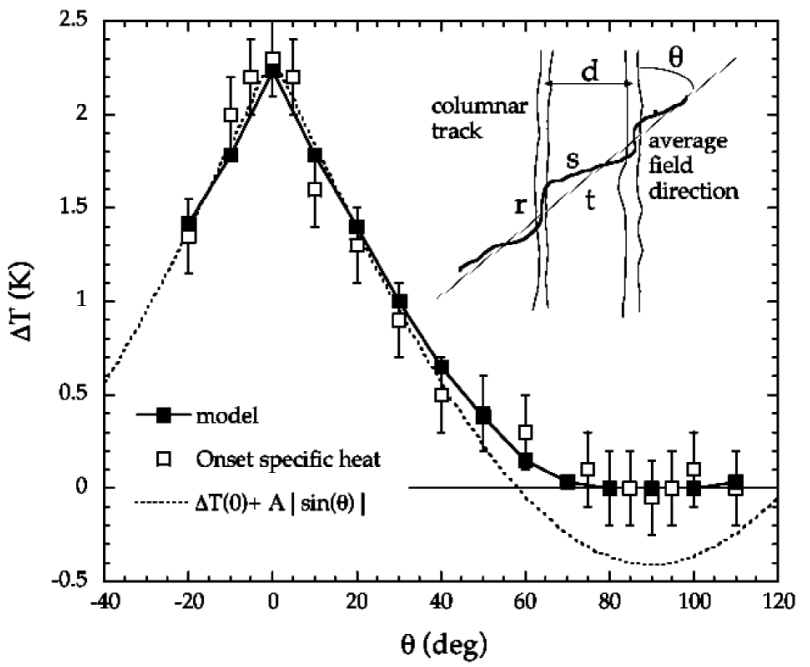

FIG. 4. Angular dependence of the transition temperature at $B=3 \mathrm{~T}$ in an irradiated $\mathrm{KBBO}$ sample $\left(B_{\Phi}=6 \mathrm{~T}\right)$ showing good agreement between experimental data (open symbols) and theoretical values deduced from Eqs. (1) and (2) (closed squares). Inset: Schematical drawing of the staircase structure formed by vortices in irradiated samples for an external field rotated with an angle $\theta$. 
Solving Eq. (1) with the new $U_{p}(\theta)$ value, one obtains the angular dependence of the transition line. The calculated values (solid squares) are presented in Fig. 4 together with the experimental values from specific heat data (open squares). Very good agreement is obtained between the experimental and theoretical values taking $U_{P} / \varepsilon_{1} \approx$ $0.7-0.8$. This corresponds to an accommodation angle $\theta_{a} \approx \arctan \left(U_{P} / \varepsilon_{1}\right)^{1 / 2} \approx 60^{\circ}-70^{\circ}$, above which the vortex staircase structure becomes unstable and vortices follow the external field. The angular dependence deduced from Eqs. (1) and (2) actually closely resembles the $|\sin (\theta)|$ law (solid line in Fig. 4) that has been predicted from scaling arguments for the Bose glass transition [12] and has been previously used as yet another argument in favor of the Bose glass melting scenario [4]. We show here that this angular dependence can also be reproduced by combining Eqs. (1) and (2).

At this point, we have shown that the shape of the transition line $\left[B^{*}(T)\right]$, its gradual increase with irradiation dose, as well as its angular dependence, can be well described if the mean-field transition temperature $T_{c}^{\mathrm{MF}} \approx$ 33 to $36 \mathrm{~K}$; irradiation then reduces the difference $\tau_{c}=$ $\left(T^{*}-T_{c}^{\mathrm{MF}}\right) / T_{c}^{\mathrm{MF}}$ for $B \neq 0$. To justify this order of magnitude, $\tau_{c}(B=0)$ can be estimated using the Brout criterion to $\tau_{c} \propto G i^{1 / 2}$ ( $G i$ is the Ginzburg number-see [13] for a discussion of the application of this criterion to high $T_{c}$ superconductors). Given the small value of $\xi$, the Ginzburg number $G i \sim 10^{-4}-10^{-3}$ is much larger than in classical superconductors and the expected shift of $T_{c}$ is therefore on the order of $0.3-1 \mathrm{~K}$. Moreover, a direct calculation of $\tau_{c}$ can also be performed within an Hartree-Fock approximation for the quartic term in the GL expansion of the free energy in the presence of a magnetic field:

$$
\begin{aligned}
\mathcal{F}_{\mathrm{GL}}=\sum_{n \mathbf{k}}[ & \left(n+\frac{1}{2}\right) \hbar \omega_{c}+a\left(T-T_{c}^{\mathrm{MF}}\right) \\
& \left.+b \sum_{n^{\prime} \mathbf{k}^{\prime}} 4\left\langle\left|\psi_{n^{\prime} \mathbf{k}^{\prime}}\right|^{2}\right\rangle\right]\left|\psi_{n \mathbf{k}}\right|^{2} .
\end{aligned}
$$

$a$ is the usual harmonic term, $b$ is the mode coupling strength, $\omega_{c}$ is the cyclotron frequency, $\psi_{n \mathbf{k}}$ is the $n$th Landau Level of the order parameter with the sums running over the wave vectors $\mathbf{k}$ in the range $\xi_{c}^{-1}<k<$ $\xi^{-1}$, and $\xi_{c}$ tis he GL correlation length evaluated at $T_{c} \sim$ $T_{c}^{\mathrm{MF}}(1+G i) . T_{c}(H)$ is obtained by setting the term within the square brackets to zero [14]. This again leads to $\tau_{c}(B=0)=2 G i^{1 / 2}$; i.e., $T_{c}^{\mathrm{MF}}-T_{c} \sim 0.6-2 \mathrm{~K}$. These estimations are therefore consistent with our model taking $\alpha$ slightly smaller than $1(\approx 0.7)$, but a more microscopic model is needed to explain the physical origin of this $\alpha$ value. Note that Eq. (3) also reproduces the strong positive curvature in $T_{c}(H)$ (dotted line in Fig. 2).
The close relationship between the thermodynamic and irreversibility lines suggests that there is no vortex liquid phase in KBBO but that the Bose glass directly sublimates into the normal state in which superconducting fluctuations still exist. Note that the critical exponents deduced from the scaling properties of the $I-V$ characteristics [7] are very similar to those predicted for the Bose glass melting. This indicates that the superconducting transition (in the presence of strong thermal fluctuations) and the Bose glass transition belong to the same universality class reflecting the fact that the normal state cannot be distinguished from the so-called entangled liquid state in which unlimited vortex loops can proliferate.

We would like to thank T. Giamarchi and D. Feinberg for very fruitful discussions.

[1] G. Blatter, M.V. Feigel'man, V. B. Geskenbein, A. I. Larkin, and V. M. Vinokur, Rev. Mod. Phys. 66, 1125 (1994).

[2] A.V. Samoilov, M.V. Feigel'man, M. Konczykovski, and F. Holtzberg, Phys. Rev. Lett. 76, 2798 (1996).

[3] C. J. van der Beek, M. Konczykowski, A.V. Samoilov, N. Chikumoto, and M.V. Feigel'man, Phys. Rev. Lett. 86, 5136 (1999).

[4] T. Klein, A. Conde-Gallardo, I. Joumard, J. Marcus, C. J. van der Beek, and M. Konczykowski, Phys. Rev. B 61, R3830 (2000).

[5] D. R. Nelson and V. M. Vinokur, Phys. Rev. Lett. 68, 2398 (1992); Phys. Rev. B 48, 13060 (1993).

[6] S. Blanchard, T. Klein, J. Marcus, I. Joumard, A. Sulpice, P. Szabo, P. Samuely, A. G. M. Jansen, and C. Marcenat, Phys. Rev. Lett. 88, 177201 (2002).

[7] C. Marcenat, S. Blanchard, J. Marcus, L. M. Paulius, C. J. van der Beek, M. Konczykowski, and T. Klein, Phys. Rev. Lett. 90, 037004 (2003).

[8] J. R. Cooper, J.W. Loram, and J. M. Wade, Phys. Rev. B 51, 6179 (1995).

[9] L. Trappeniers et al., Physica (Amsterdam) 313C, 1 (1999); C. J. van der Beek et al., Phys. Rev. B 61, 4259 (2000).

[10] I. Joumard, J. Marcus, T. Klein, and R. Cubitt, Phys. Rev. Lett. 82, 4930 (1999).

[11] The IRL lies below the onset of the specific heat anomaly and slightly different parameters: $\xi \sim 35 \AA$ and $\lambda \sim$ $3300 \AA$ have been used in this simulation.

[12] M. Wallin and S. M. Girvin, Phys. Rev. B 48, R6845 (1993); J. Lidmar and M. Wallin, Europhys. Lett. 47, 494 (1999).

[13] A. Kapitulnik et al., Phys. Rev. B 37, 537 (1988).

[14] See B. I. Halperin and D. R. Nelson, J. Low Temp. Phys. 36, 599 (1979) for a similar approach in superconducting films. 